\title{
Choix du débit de crue pour lequel il convient de prévoir les ouvrages évacuateurs d'un barrage
}

\section{Choice of the flood discharge for which the spillway structures of a dam should be designed}

\author{
PAR .J. DUFFAUT
}

\author{
INGENIEUR EN CHEF DES PONTS ET CHAUSSELS \\ CHEF DU SEIRVICE TEGHNIQUE DE L'ENERGIE ELECTRIQUE ET DES GRANDS BARMAGES
}

\begin{abstract}
Impossibilité de se faire une idée exacte de l'importance et de la fréquence, dans le temps. des crues exceptionnelles. Lauteur propose de substituer à la notion de fréquence dans le temps la notion de fréquence dans l'espace. Si au lieu de cuelques grands bassins, on considèrc une multitude de petits bassins, l'expérience montre que l'on peut en quelques années enregistrer de véritables cataclysmes hydrologiques qui, pour être localisés, n'en peuvent pas moins donner des indications précieuses sur les "plus grandes crues possibles $»$.
\end{abstract}

L'importance du choix d'un débit de crue assez élevé pour que le barrage ne soit pas surmonté provient du danger que court tout barrage lorsqu'il est surmonté. Les barrages peuvent périr en effet sous l'action de diverses causes, mais l'expérience montre que l'insuffisance des ourrages d'évacuation des crues a été dans le passé une cause très importante.

D'autre part, on assimile parfois la montée de l'eau due à la rupture d'un barrage à une crue naturelle. Il est pourtant bien évident qu'il $y$ a entre les deux une différence considérable.

Dans une crue naturelle, l'eau monte d'une facon relativement lente et régulière et la crue est́ précédée de phénomènes météorologiques qui la font prévoir à supposer qu'elle ne fasse pas l'objet d'avis donnés à l'avance par le Service d'annonce des crues.

Maloré ces conditions favorables, les crues naturelles font fréquemment des victimes et provoquent parfois de véritables catastrophes comme à Toulouse en 1875 ou à Moissac en 1930.

Par contre, la crue provoquée par la rupture d'un barrage est bien plus destructrice parce cu'elle pent atteindre des hauteurs beaucoup plus élevécs cule celles'des crues naturelles et ce, avec une rapidité incomparahlement plus grande.

D'autre part, lorsque des populations s'établissent ou restent dans une zone submersible en temps de sirandes crues, elles le font volontairement el dans une certaine mesure en connaissance de cause.

Au contraire, lorsqu'on établit un harrage
The accurate determination of the magnitude and frequency, in time, of exceplional floods, is an impossible matter. The anthor proposes to substitute the notion of frequency in space for the notion of frequency in time. Experience shows that, a great number of small watersheds being considered instead of a few large ones, veritable hydrologic catastrophes can be observed within a few years; these catastrophes, although occuring in restricted areas, can give valuable information on the "maximum floods".

d'assez grande hauteur, sur un cours d'eau, on suspend au-dessus des riverains aval, sans qu'ils puissent pratiquement s'y opposer, la menace permanente d'une crue de hauteur comparable à celle du barrage (du moins à son voisinage), c'est-à-dire bien plus destructrice que les crues naturelles du cours d'eau qui atteignent des hauteurs beaucoup moindres et beaucoup moins vite.

Il suffit d'ailleurs de rappeler les ruptures de barrages les plus récentes : Molare, San Francis, Gleno, Perrégaux, pour évoquer de véritables catastrophes.

Les barrages sont donc des ouvrages d'art que l'ingénieur doit s'efforcer d'édifier pour l'éternité.

Malheureusement, il ne paraît pas possible d'assigner avec précision un maximum indépassable au débit des crues d'un cours d'eau en un point déterminé.

Sans doute, personne n'imagine qu'un bassin versant de 1 . $\mathrm{km}^{2}$ par exemple, pour lequel on envisagera sans doute au maximum une crue de 60 à $70 \mathrm{~m}^{3}$ par seconde, peut présenter une crue de $10.000 \mathrm{~m}^{3}$ par seconde. Mais une crue de 600 à $700 \mathrm{~m}^{3}$ y est-elle impossible?

On ne saurait répondre affirmativement lorsqu'on voit attribuer des débits de l'ordre de 40 à $50 \mathrm{~m}^{3} / \mathrm{s}$ par $\mathrm{km}^{2}$ à des bassins versants du même ordre de grandeur au cours de la crue qui s'est produite en octobre 1940 dans le haut hassin du Tech (Pyrénées-Orienlales).

En fait, sur des bassins de cet ordre, on n'a jamais prévu les ouvrages d'évacuation pour un 
debil de plus de $10 \mathrm{~m} \% / \mathrm{s} / \mathrm{km}^{2}$ environ, qui est déjà très élevé.

On voit ainsi que la considération d'un débit maximum est sans objet pratique parce que si un tel débit existe certainement, d'mne part on ne peut pas le déterminer avec précision, d'autre part il est tellement élevé qu'on ne peut songer en aucun cas, pour des raisons économiques, à clablir des ouvrages pour un débit aussi grand.

De celte impossibilité découle gue tout barrage fait courir un risque inévitable aux populations siluées à l'aval. Ce risque tient pour une large part à la cause particulière de ruine que représcnte un débit supérieur au débit naximum pour lequel les ouvrages d'évacuation sont établis.

On doit donc choisir ce dernier de façon que les chances de retour d'une rupture de barrage soient asser faibles sur l'étendue du territoire métropolitain. Nous pensons que cette période moyenne de retour devrait être de l'ordre de cinquante ans ${ }^{1}$ au moins pour être supportable. Dès lors, si on estime à 200 environ le nombre de barrages francais (y compris ceux qui sont en construction et tous ceux qui restent à construire) dont la rupture serait très dangereuse, on est conduit à retenir comme débit de crue celui qui se produit tous les :

$200 \times 50=10.000$ ans environ.

Le choix de la durée de cinquante ans ci-dessus est, bien entendu, essentiellement subjectif. On peut penser qu'une durée plus faible serait supportable.

Mais il faut tenir compte qu'il ne s'agit pas d'une période véritable mais d'une moyenne, qui n'exclut nullement deux catastrophes successives beaucoup plus rapprochées. Dans ce dernier cas, la réaction du public serait (à tort sans doute, mais à coup sûr) beaucoup plus vive.

Ce qui importe d'ailleurs au point de vue psychologique ce sont les effets, c'est-à-dire la ruine de l'ouvrage et non les causes. L'insuffisance des ouvrages d'évacuation des crues, qui n'est qu'une de ces causes, doit donc être moins fréquente que la ruine d'un ourrage. En outre, étant donné la proximité géographique d'un certain nombre d'ouvrages, il n'est pas douteux que deux ou plusieurs d'entre eux peuvent être affectés par le même phénomène météorologique exceptionnel. L'insuffisance des ouvrages d'évacuation des crues peut ainsi apparaître sur plusieurs ouvrages à la fois.

Enfin, on se trouve de plus en plus en présence d'ouvrages en série sur le même cours d'eau ou ses affluents. C'est ainsi par exemple que la seule vallée de la Dordogne, en amont d'Argentat, ne comporte pas moins de dix barrages qui intéressent fortement la sécurité pu-

1. La dernière ruplure de barrage en France est celle de Bouzey en 1895. blique. Qu'arriverait-il aux harrages aval si un des barrages amont venait à se rompre? Il $\mathrm{y}$ aurait à craindre évidemment d'autres ruptures comme conséquence de la première.

Les considérations précédentes tendraient à faire considérer comme désirable une durée moyenne de plus de cinquante ans entre la ruine de deux harrages successifs.

Fort heureusement, une autre considération fort importante joue en sens inverse. Un barrage surmonté n'est pas nécessairement un barrage détruit. Seul un barrage en terre ne peut supporter aucun déversement. Il n'est pas douteux que la plupart des barrages en maconnerie francais pourraient en supporter un sans céder. Un tel déversement pourrait causer d'importants dégâts notamment à l'usine lorsqu'elle est située contre le barrage, mais il ne s'agirait que de dégâts matériels dont le public ne souffrirait pas et ne s'apercevrait même pas. La durée de cincuante ans admise plus haut devrait donc être multipliée par la probabilité pour que l'insuffisance des évacuateurs de crues entraine la ruine du barrage. Difficile à apprécier, cette probabilité n'est peut-être que d'un quart on d'un tiers.

Si on voulait tenir compte de cette considération favorable, en faisant abstraction des considérations défavorables énoncées antérieurement, on serait conduit à envisager la crue qui peut se produire en moyenne une fois tous les 2.500 à 3.000 ans $^{2}$.

\section{Débit de la plus grande crue connue}

Fn dehors de toute question de durée de retour, le débit de la plus grande crue connue ne peut naturellement être ignoré lorsqu'on a à prévoir le débit de crue à adopter pour un ouvrage.

Mais quel usage doit-on faire de ce débit?

En général on en tient compte en prévoyant les ouvrages pour un débit au moins égal et souvent on multiplie le débit de la plus grandc crue connue par un coefficient de sécurité.

Cette facon de faire, cui pent paraitre naturelle, n'est pas logique à la réflexion.

Ia période de retour de la plus grande crue connue est en effet indéterminée de par sa nature même et peut être bien plus grande cue celle, estimée plus haut à 10.000 ans, pour laquelle il paraît logique de calculer les ouvrages d'évacuation des crues.

C'est le cas probablement pour la crue d'octobre 1940, dans le bassin du Tech, pour laquelle M. PAndé admet les débits de 12 à

2. Cette durée correspond à peu près à celle de 2.750 ans admise, pour d'autres raisons, par M. A. CouTAGNe dans son rapport au $4^{\mathrm{e}}$ Congrès des Grands Barrages. 
$13 \mathrm{~m}^{3} / \mathrm{s} / \mathrm{km}^{2}$ et de 9 à 10 pour $350 \mathrm{~km}^{2}$. Fn effet, en ajustant les débits du Tech à Prats-de-Mollo (B.V. 76, $6 \mathrm{~km}^{2}$ ) suivant la loi de M. GimRat, on ne trouve qu'un débit de $1.865 \mathrm{l} / \mathrm{s} / \mathrm{km}^{2}$ pour une période de retour de 10.000 ans ". Mais qui prendra la responsabilité d'adopter un débit inférieur à celui de la plus grande crue connue? Personne ne s'y risquera, pensons-nous.

Aussi, croyons nous inévitable, et somme toute raisonnable, de conserver la règle de prévoir un débit de crue supérieur ou égal à celui de la plus grande crue observée.

\section{Détermination d'un débit de crue de probabilité donnée}

Il serait facile de déterminer pratiquement un débit de crue de probabilité donnée si on possédait pour l'endroit considérć plusieurs dizaines de milliers d'années d'observations. Tout lo monde admettrait en effet, pensons-nous, que la fréquence d'un débit déterminé resterait, au cours des années à venir, la même que celle qui résulterait des observations passées.

Mais, en fait, on est extrêmement loin do cette situation.

On ne possède pour les grandes crues que des données portant sur quelques centaines d'années pour les grands bassins versants, là où la présence d'une ville importante telle que Paris. Lyon, Toulouse, a conduit à redouter les crues depuis très longtemps et à en relever les traces. Encore les débits sont-ils, même à ces endroits favorisés, beaucoup moins connus que les hanteurs.

De lincertitude du débit des grandes crues sur les grands bassins, nous donnerons les deux exemples suivants :

Sur la Dordogne, à Argentat par exemple, le débit de la crue de 1783 peut être estimé à $2.630 \mathrm{~m}^{3} / \mathrm{s}$ ou à près de $4.000 \mathrm{~m}^{3} / \mathrm{s}$, suivant la façon dont on extrapole la courbe des débits en fonction des hauteurs d'ean ${ }^{4}$.

Sur le Tarn, à Pinet, le calcul de la crue maxima connue en ce point, celle de septembre 1875, a été effectué en 1924 en appliquant la formule du mouvement graduellement variè à des profils en travers exactement relevés avec un coefficient de débit vraisemblable et pour des cotes maxima atteintes, admises d'après la tradition. Suivant les diverses sections du cours d'eau considérées, on arrivait ainsi à des valeurs

3. Il est vrai que cela correspondrait à une durce de 24 heures pour le débit de $1.865 \mathrm{l} / \mathrm{s}$ alors que le débit maximum de la crue de 1940 a duré tout au plus quelques dizaines de minutes.

4. Voir Monographie Hydrologique de la IIante-Dordogne, par MM. Jacques Tissirr et Ernest Zaccagino. Annuaire hydrologique de la France. Annéc 1944. du débit comprises entre 2.000 et $5.000 \mathrm{~m}^{3}$ environ. On adopta pour le barrage de Pinet la valeur moyenne de $3.500 \mathrm{~m}^{3}$, soit $1.300 \mathrm{l} / \mathrm{s}$ par $\mathrm{km}^{2}$ de bassin versant, bien qu'elle fût incertaine à $40 \%$ près environ.

Quant aux crues exceptionnelles qui affectent les petits bassins versants, elles ne sont généralement pas observées, ni enregistrées, soit parce qu'aucune personne compétente n'en est témoin, soit, surtout, parce que les dégâts causés sont généralement faibles.

A cet égard, il faut observer que ce n'est naturellement pas au cours d'une crue considérée comme exceptionnelle sur un bassin versant de 5.000 ou $10.000 \mathrm{~km}^{2}$ qu'on observera la crue exceptionnelle d'égale probabilité sur un bassin versant de $100 \mathrm{~km}^{2}$ compris dans le grand bassin précédent.

La crue exceptionelle d'un petit bassin versant est due à un phénomène météorologique très limité dans l'espace et dans le temps qui intéresse spécialement ce bassin versant. Tout à fait exceptionnelle sur te petit bassin versant, une crue de ce gence s'atténue tris rapidement notamment dès qu'elle se jette dans un cours d'eau plus important.

Sur celui-ci, on n'a plus qu'une crue très modérée ou même négligeable.

Une telle crue passe donc inapercue ou n'est même pas considérée comme digne d'être enregistrée aux endroits où l'observation des hauteurs de crue est assurée avec soin et depuis longtemps à cause des dégâts qui peuvent en résulter.

C'est chaque année, nous en sommes persuadé, qu'au cours notamment des périodes orageuses, des débits dépassant le mètre cube par seconde et par $\mathrm{km}^{2}$ et atteignant peut-être $2,3 \mathrm{~m}^{3} / \mathrm{s} / \mathrm{km}^{2}$ ou même davantage, se produisent sur des bassins versants de l'ordre de quelques kilomètres carrés ou de quelques dizaines de kilomètres carrés, sans que ces phénomènes, pourtant exceptionnels, soient enregistrés.

Pendant la construction du barrage de Marcges, nous avons observé ainsi avec stupéfaction une crue extrêmement violente due au ruisseau d'Enval, affluent de la Dordogne à Marèges, crue au cours de laquelle ce ruisselet, dont le hassin versant est de l'ordre de $5 \mathrm{~km}^{2}$, a déplacé des matériaux solides au point de recouvrir en quelques instants une pelle à vapeur occupée à exécuter les foulles du barrage dans le lit du ruisseau. Cette crue serait restée absolument ignorée si les travaux du barrage de Marèges n'avaient pas été en cours à ce moment-là.

Plus près de nous, le 16 juin 1951, une crue extrêmement violente s'est produite sur la Seuge, affluent rive gauche de l'Allier, dans Icquel clle se jette à Prades, à quelques kilomettres en amont de Langeac (Haute-Loire). 
En l'espace de deux heures environ, ce cours d'eau, qui débitait tout au plus 2 ou $3 \mathrm{~m}^{3} / \mathrm{s}$, est passé à un débit que nous avons estimé finalement à $305 \mathrm{~m}^{3} / \mathrm{s}$ environ pour un bassin versant de $122 \mathrm{~km}^{2}$, soit $2,5 \mathrm{~m}^{3} / \mathrm{s}$ et par $\mathrm{km}^{2}$. La présence d'un barrage déversant d'une dizaine de mètres de hauteur et le relevé précis du niveau atteint par la Seuge à l'amont du barrage permettent d'affirmer que ce débit extraordinaire doit être très près de la réalité.

C'est uniquement aux dégâts très sérieux qu'elle a causés (une dizaine de maisons détruites à Prades, six ponts emportés) que cette crue a dû de retenir l'attention.

Il convient de noter, en outre, que la mesure précise du débit n'a été possible que grâce à la présence du barrage de Luchadou. La station de jaugeage située en amont n'aurait pu donner qu'une idée très vague de l'importance de la erue.

Devant l'insuffisance du nombre d'observations au point considéré pour déterminer le débit de la crue en ce point, nous avons attiré lattention, dans notre rapport sur la question

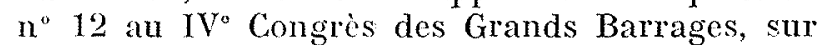
la possibilité de pallier cette insuffisance en multipliant les observations dans l'espace de façon à obtenir en peu de temps beaucoup dannées d'observations grâce à des stations plus nombreuses. Il y a là une possibilité rapide d'améliorer notre connaissance des débits de crue sur laquelle nous croyons devoir insister.

A l'heure actuelle, par exemple, notre Service exploite une centaine de stations de jaugeage dans les bassins de la Dordogne, de l'Allier, de la Loire en amont du confluent de l'Allier, sur la Loire en aval de ce confluent, sur ses affluents rive gauche et sur les fleuves côtiers entre Loire et Garonne.

Voici comment se répartissent ces stations suivant l'étendue du bassin versant :

Bassins versants en $\mathrm{km}^{2} \quad$ Nombre de stations

10 a $100 \mathrm{~km}^{2} \ldots \ldots \ldots \ldots \ldots \ldots \quad \overline{26}$

100 à $1.000 \mathrm{~km}^{2} \ldots \ldots \ldots \ldots \ldots \ldots .55$

1.000 à $10.000 \mathrm{~km} \mathrm{~km}^{2} \ldots \ldots \ldots \ldots \ldots .617$

Plus de $10.000 \mathrm{~km} 2 \ldots \ldots \ldots \ldots . .1$

Total............ 99

Voici également comment se répartissent, suivant l'étendue du bassin versant, les 25 barrages principaux existant dans notre Service ${ }^{\check{2}}$ :

5. Ces barrages constituent d'excellentes stations de jaugeage pour les débits de crue. On peut dire que les débits de crues ne commencent à être bien connus en un point qu'ì partir du moment où on $y$ a ćtabli un barrage.
Bassins versants en $\mathrm{km}^{2} \quad$ Nombre de barrages

Moins de $10 \mathrm{~km}^{2} \ldots \ldots \ldots \ldots \ldots$ - 1

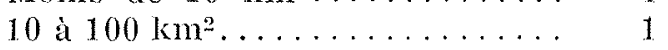

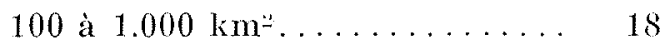

1.000 ¿ $5.000 \mathrm{~km}^{2} \ldots \ldots \ldots \ldots \ldots .5$

Total............ 25

Le tableau précédent montre l'importance dominante, tout au moins dans notre région, en matière d'aménagements hydro-électriques, des bassins versants compris entre 100 et $1.000 \mathrm{~km}^{2}$.

Il est bien évident qu'on pourrait multiplier les stations de jaugeage sur les bassins versants de 10 à $100 \mathrm{~km}^{2}$, qui existent naturellement on nombre plus grand que ceux de 100 à 1.000 . Il ne faudrait pas croire toutefois qu'il y en a dix fois plus.

Dans un bassin versant de $1.000 \mathrm{~km}^{2}$ on ne trouve pas, en effet, 100 bassins versants indépendants de $10 \mathrm{~km}^{2}$, mais seulement une trentaine, si on ne retient comme bassins versants indépendants que ceux dont toute l'eau s'écoule par un seul émissaire et qui ne comportent aucun affluent. Des mesures sur les bassins versants qui ne répondent pas à cette définition ct qui couvrent le reste de la surface considérée sont théoriquement possibles, mais leur précision serait faible étant donné la nécessité d'opérer par différence.

Sans doute, pour pouvoir tirer des observations faites sur de nombreux bassins des conclusions valables pour chacun d'eux, il convient que tous les bassins versants élémentaires puissent être regardés, sinon comme absolument identiques, du moins comme suffisamment semblables en ce qui concerne les conditions de production des grandes crues. Ces conditions dépendent, nolamment, comme on sait, de la forme du bassin, du relief, de la nature géologique du sol, du boisement et des cultures, mais elles dépendent aussi, d'une facon tout à fait dominante, des conditions météorologiques. Il ne faut donc comparer autant que possible que des bassins soumis à peu près aux mêmes conditions pluviométriques ${ }^{6}$.

Mais même si les bassins observés ne sont pas identiques à ce point de vue, il n'est pas douteux que l'extension des observations à de nombreux bassins permettrait d'acquérir assez rapidement des connaissances plus précises sur la fréquence des débits de crue pour les petits bassins versants.

On pourrait ainsi par exemple dans notre Ser-

6. Malheureusement on ne dispose a cet égard que de renseignements insuffisants. On n'a pas notamment pour chaque rëgion des tableaux dommant l'intensité des averses en fonction de leur durée et de la surface à laquelle elles s'étendent. 
vice majorer de quelques dizaines le nombre des stations de jaugeage dont le bassin versant est compris entre 100 et $1.000 \mathrm{~km}^{2}$ et de quelques centaines peut-ètre les stations dont le bassin versant est compris entre 10 et $100 \mathrm{~km}^{2}$. En dix ans seulement, on augmenterait ainsi pour ces dernières de plusieurs milliers le nombre total d'années d'observation, ce qui permettrait la détermination de la fréquence moyenne de débits déjà assez rares.

Nous avons rapproché dans cet esprit en 1950 les résultats fournis par 28 stations de jaugeage de notre Service dont nous avons considéré les bassins versants (à tort ou à raison) comme suffisamment semblables. Ainsi, bien qu'aucune de ces 28 stations n'ait été exploitée pendant plus de 33 ans, nous sommes arrivés pour l'ensemble de ces stations à 750 années d'observations environ au cours desquelles on a relevé :

$\begin{array}{cccll}1 & \text { débit instantané } & \text { de } 1.500 \mathrm{l} / \mathrm{s} / \mathrm{km}^{2} \tau \\ 1 & -- & - & \text { de } 1.100 \quad- & \\ 1 & - & - & \text { compris entre } 900 \text { et } & 1.000 \\ 2 & \text { débits instantanés } & \text { compris entre } 800 \text { et } & 900 \\ 5 & -- & - & \text { compris entre } 700 \text { et } & 800 \\ 15 & - & - & \text { compris entre } 600 \text { et } & 700 \\ 14 & - & - & \text { compris entre } 500 \text { et } & 600\end{array}$

On peut en conclure, pensons-nous, avec quelque sécurité, que, sur les bassins versants intéressés, les débits de 700 à $800 \mathrm{l} / \mathrm{km}^{2}$ ont une probabilité de se produire d'environ $\frac{5}{750}=\frac{1}{150}$ que cette probabilité est d'environ $\frac{15}{750}=\frac{1}{50}$ respectivement pour les débits de 600 à 700 litres et pour ceux de 500 à 600 litres.

Par contre, il n'est pas possible d'apprécier la probabilité d'un débit individuel supérieur à 800 litres. Tout au plus peut-on dire, puisque 5 débits dépassent 800 litres, que la probabilité de voir dépasser ee dernier débit est de l'ordre de $\frac{1}{150}$ environ comme celle d'observer un débit compris entre 700 et 800 litres.

On pourrait dire également que la probabilité d'avoir un débit compris entre $\$ 00$ et 1.000 litres est de l'ordre de $\frac{3}{750}=\frac{1}{250}$ mais sans pouvoir en tirer un parti bien précis car la probabilité est moins approchée que les précédentes et est relative à un intervalle de débit plus grand.

Quant au débit maximum de 1.500 litres, qui est nettement isolé de l'ensemble des autres observations, il est impossible, en se basant seulement sur l'expérience, de dire quoi que ce soit de la probabilité à laquelle il correspond.

7. Un débit de $2.500 \mathrm{l} / \mathrm{s} / \mathrm{km}^{2}$ a été observé en $1951 \mathrm{sur}$ la Seuge comme il est indiqué plus haut.
Cela est extrêmement fâcheux car ce débit maximum nous intéresse tout particulièrement.

Pour essayer d'aller plus loin, il faut faire appel à une des formules qui prétendent indiquer, même pour les très faibles probabilités, comment varie le débit en fonction de la probabilité.

En prenant pour cela une formule quelconque, par exemple celle de Furrar, e'est-à-dirc le coefficient $(1+0,8 \log \mathrm{T})$, on reconnait que pour ${ }^{~} \mathrm{~T}=150$ ce facteur a une valeur de 2,74 et pour $\mathrm{T}=250$ une valeur de 2,92 , alors qu'il a une valeur de 4,2 pour $\mathrm{T}=10.000$ ans.

Dans ces conditions, on aurait une chance de voir se produire en 10.000 ans un débit compris entre:

$$
\begin{aligned}
700 \times \frac{4,2}{2,74} & =1.070 \mathrm{l} / \mathrm{s} / \mathrm{km}^{2} \\
\text { et } 800 \times \frac{4,2}{2,74} & =1.220 \mathrm{l} / \mathrm{s}^{\prime} / \mathrm{km}^{2}
\end{aligned}
$$

et une chance égale de voir ce dernier débit dépassé, mais sans savoir de combien.

On aurait de même une chance de voir se produire en 10.000 ans un débit compris entre :

$$
\begin{array}{r}
800 \times \frac{4,2}{2,92}=1.150 \mathrm{l} / \mathrm{s}^{-\mathrm{km}^{2}} \\
\text { ct } 1.000 \times \frac{4,2}{2,92}=1.440 \mathrm{l} / \mathrm{s} / \mathrm{km}^{2}
\end{array}
$$

Le débit de 1.500 litres, pourtant observé uno fois, apparaîtrait ainsi comme un phénomene isolé dont la probabilité d'apparition serait sans doute inférieure à une fois en 10.000 ans.

Mais les bassins versants considérés n'ayant pas tous les mèmes caractéristiques et la validité de la formule de Fuluer étant incertaine, ainsi d'ailleur's que les relations hauteur-débit des stations considérées, il est prudent de se prémunir contre des débits de crue nettement supérieurs aux chiffres indiqués ci-dessus.

\section{Conclusions}

Le débit de crue maximum maximorum, e'estì-dire de probabilité rigoureusement nulle, ne peut être déterminé avec précision.

Il est beaucoup plus grand (cinc à dix fois plus peut-être) que les débits pour lesquels on établit d'habitude les ouvrages d'évacuation des crues des barrages.

Ceux-ci étant déjà très onéreux, il apparaît en général économiquement impossible d'établir ces ouvrages pour le débit maximum maximorum. On est ainsi amené à adopter des débits de crue dont la probabilité d'apparition est très faible sans être nulle. 
Quelle probabilité adopter?

En considération du nombre de barrages existants ou à créer dont la rupture causerait de graves dommages, il paraît raisonnable de se prémunir en France contre un débit de crue dont la probabilité de retour est de 10.000 ans environ. Malheureusement, les observations disponibles portant sur une période beaucoup plus courte, le débit qui présente cette probabilité ne peut être déterminé d'après les résultats statistiques. On peut alors recourir aux formules proposées par divers auteurs, mais leur application pour des probabilités très faibles constitue une extrapolation hasardeuse particulièrement pour les bassins versants de moins de $1.000 \mathrm{~km}^{2}$ à débit très irrégulier.

D'autre part, on considère partout comme un élément fondamental dans le choix du débil de crue à évacuer le débit de la plus grande crue connue. Ce débit, difficile souvent à déterminer avec précision, est également difficile à utiliser, car le fait qu'il est unique ne permet pas de reconnaître à quelle probabilité de retour il correspond. On ne sait de combien il faut le corriger pour obtenir le débit correspondant à la probabilité admise. Dans cette ignorance, il paraît raisonnable d'adopter toujours un débit au moins égal à celui qu'on attribue à la plus grande crue connue.

Il apparaît ainsi en fin de compte, faute de pouvoir déterminer avec précision le débit correspondant à la probabilité admise et la probabilité du plus grand débit connu, qu'il n'est pas possible de résoudre de façon rigoureuse le problème posé. On est obligé en pratique de prendre un risque sans même pouvoir en déterminer exactement l'importance.

D'où l'intérêt de prévoir les ouvrages avec des dispositions telles que, si le débit de crue admis vient à être dépassé (ce qui arrivera, inévitablement un jour ou l'autre pour tel ou tel barrage), il n'en résulte autant que possible pour l'ouvrage que des dégâts mineurs et non la ruine complète. On est ainsi conduit à adopler en un mème point des débits de crue sensiblement plus élevés pour un barrage en terre que pour un barrage en béton ef pour un ivacuateur de erue en charge que pour un évacuateur de crue superficiel.

Le défaut d'observations dans le temps pourrait être pallié, dans une certaine mesure, par une extension des observations dans l'espace. S'il n'existe en France que 15 ou 20 bassins de plus de $10.000 \mathrm{~km}^{2}$, on $\mathrm{y}$ trouve environ 1.500 a 2.000 bassins de $100 \mathrm{~km}^{2}$. Dix années seulement d'observations complètes sur tous ces bassins pourraient être regardées dans une certaine mesure comme équivalentes à 15.000 à 20.000 années d'observations sur un seul. On pourrait en déduire par conséquent très vite, sans recouxs à des formules de valeur douteuse, le débit correspondant approximativement à la probabilité d'une apparition par dix mille ans admise précédemment.

Bien entendu, on aurait ainsi un débit moyen à corriger d'après les conditions particulières de chaque bassin.

D'autre part, on n'aurait un renseignement assez précis que pour un bassin versant de l'ordre de $100 \mathrm{~km}^{2}$. Mais comme on a tout de même déjà une idée relativement bonne de la probabilité de la crue centenaire sur les grands bassins (de plus de $10.000 \mathrm{~km}^{2}$ par exemple), on pourrait, par interpolation entre petits et grands bassins versants, déterminer avec quelque sécurité les débits de crue à adopter pour les bassins versants de taille intermédiaire.

On est malheureusement très loin d'avoir des stations de jaugeage sur tous les bassins versants distincts de $100 \mathrm{~km}^{2}$ environ (à peine le dixième sans doute). D'autre part, les stations existantes sont la plupart limitées à la détermination des débits de hautes eaux et ne se prêtent généralement pas à la détermination tant soit peu précise d'un débit exceptionnel et fugitif.

La possibilité intéressante qu'ouvrirait l'extension du nombre de stations de jaugeage sur les petits bassins versants exigerait donc des dépenses importantes qu'il faudrait sans doute échelonner et, par suite, une longue continuité de vues. Nous estimons néanmoins très désirable de s'engager dans cette voie pour contribuer à la solution du probleme du débit de crue à choisir pour un barrage.

\section{I S C U S S I O N}

M. le président distingue et souligne deux idées fondamentales dans la communication de M. Durfaut :

10 Le choix d'une valeur limite comme fréquence des catastrophes ou période de retoux (50 ans) au-dela de laquelle l'émotion des populations deviendrait critique : cóté psychologique du problème des crues qui diffère du cóté économique développé à New-Delhi par M. Dansi. lequel mettait en balance l'aceroisscment de sécurité et la depense supplémentaire qui resulterait de cet accroisscment;

2" La substitution de la multiplicité des données dans l'espace a l'ancienneté des données dans le temps, lorsque celle-ci fait défaut pour l'étude statistique des phènomènes.

Dans quelle mesure peut-on admettre cette substitution?

M. Mary pense que dans certains cas particuliers, il est dangereux de prévoir les évacuateurs de crues pour des débits supérieurs à coux que la rivière elle-même peut écouler à l'aval; dans le cas où le barrage lui-même pourait subir sans rupiure des déversements (barrages, vôtes, ou barrages mixtes poids-voute), l'éventualité 
d'un tel déversement est justiciable d'un calcul d'assurance dans le sens qui a été indiqué par M. Daner.; en particulier, si l'usine se trouve au pied du barrage, elle peut subir des dégâts importants mais la prime d'assurance ne correspond certainement pas à une probabilité très faible des débits des crues. Par contre, un excès de dimensionnement des évacuateurs de erues peut provocuer des dégats considérables dans la vallée et il suffit d'une fausse mancuvie ou d'un acte de guerre (bombardement ou sabotage) pour provoquer une catastrophe.

M. Ie Président souligne que la pratique critiquée par M. ManY est en effet susceptible d'accroître la responsabilité de l'exploitant du barrage.

M. Durrayt est de cet avis; toutefois, l'erreur, selon lui, n'est pas de prendre un débit qui peut paraître trop éleve, mais de le concentrer sur un trop petit nombre d'engins, ce qui est très dangereux pour l'ouvrage en cas de panne et pour l'aval en cas de fausse manouve.

Cependant, M. Mary estime que la vulnérabilité de louvrage n'est guère réduite par la multiplication des cvacuateurs, et qu'elle tient surtout au fait que les vannes qui commandent ces évacuateurs sont plus faciles it démolir que le barrage lui-mème.

M. Chamayou considere que le risque d'une catastrophe, qu'il soit basé sur une probabilité donnée de crue ou sur une frequence de rupture du barage, suppose que l'événement se produit à réservoir plein, ce qui n'est réalisé, le plus souvent, que pendant une fraction plu: ou moins grande de l'année, pouvant descendre jusqu'a 15 jours par an pour certains réservoirs (Caillaous, Portillon, Bouillouses). En toute autre période, le réservoir est généralement capable d'absorber la crue. Le risque de déversement ou d'évacuation catastrophique est done réduit de ce fait et doit être basé sur une probabité compose prenant en compte la frécuence du remplissage complet du réservoir.

M. Dufraur reconnait que l'observation de M. CHAsayou est fondee, mais seulement pour les barragesréservoirs.

M. Serna observe que l'on pourrait aussi inclure dans te calcul du risque la probabilite des averses pendant les périodes de remplissage, et dans la région où se trouve le barrage.

M. Fon'ane remarque que les conditions des crues millénaires sont multiples et que leur coincidence est exceptionnelle et propre à certaines zones bien délimitées.

M. Durfaut indique que son étude est basée sur des phénomènes observés généralement dans l'ouest du Massif Central : affluents rive gauche de la Loire supérieure, bassin de la Dordogne, plateau de Millevaches. Ces phénomènes sont, selon toute probabilité, encore plus accentués dans certaines régions voisines, comme la bordure orientale du Massif Central (Vivarais et Cévennes). On a observé en novembre dernier $1.800 \mathrm{l} / \mathrm{s}$ par $\mathrm{km}^{2}$ sur la Loire supérieure a Lapalisse et sur la Fontaulière. Une comparaison d'observations pluviométriques s'étendant sur 20 années de part et d'autre de la chaîne des Cévenmes serait certainement intéressante.

M. Pardé remarque à ce sujet que dans le bassin de l'Ardèche, qui n'a pas eu de vraies très fortes crues depuis cinquante ans, de petites surfaces réceptrices ont cébité un grand nombre de fois $2 \mathrm{~m} 3 / \mathrm{s}$ et plus par $\mathrm{km}^{2}$; répondant a une question de M. Fontaine, il explique que la distribution des crues, d'où l'on déduit la fréquence moyenne, peut n'être vérifiable qu'à l'échelle de 1.000 ou 10.000 ans (exemple: la Garonne à Toulouse où aucune grande crue n'a été observée depuis le cataclysme de 1875). D'autre part, il peut $y$ avoir répétition des phénomenes exceptionnels sur de courtes périodes, soit que la diversité des facteurs en accroissent la probabilité, comme cela pourrait être le cas de la Garonne a Agen en mars 1927 , en 1930 et 1935 ; soit que leurs causes se répètent elles-mêmes par l'efret du hasard comme on peut le supposer pour les crues de l'Inn en 1598 et 1606 , puis en 1786 et 1787 , faute d'autres explications.

Pour ce qui concerne la meillcure utilisation de l'ensemble des séries d'observations dont on peut disposer dans une région donnée, M. MonLat soumet deux suggestions :

$1^{\circ}$ Comme l'a souligné M. Dufraut, l'utilisation simultané des crues annuelles observées pendant trente ans sur trente cours d'eau du Massif Central, par exemple, n'est pas équivalente a neut siècles d'observations sul un seul de ces bassins, du point de vue de l'information apportée pour la loi de probabilité des crues. On est, en cfiet, en présence du dilemme suivant : ou bien on groupera des stations de régimes aussi semblables que possible, et alors les crues observées sont très loin d'être indépendantes; lo gain d'information lisque d'être faible par lapport à l'emploi de la seule station étudiée; - ou bien on groupera des stations de régimes hétérogènes, et les crues observées pourront mieux être considérées comme des phénomènes distincts, mais il n'y a généralement pas de raisons d'admettre alors que les crues survenues sur ces divers bassins obeissent aux mèmes tois, et l'interprétation des résullats, quant aux crues d'un bassin particulier, scra fort difficite.

En fait, M. Duffaut a applique la methode a des bassins relativement lomogènes du Massif Central. Dans ce cas, on ponrmit avoir une idée da gain d'information qu'apporte ce procédé en admettant qu'on obtient en fin de compte autant d'observations qu'il y a de crues annuelles survenant $\mathbf{a}$ des dates nettement distinctes.

On négligerait encore les facteurs généraux du climat. et on ne peut affirmer que l'ensemble des observations de trente stations, portant chacune sur trente ans, soit equivalent à cent cinquante ans si on a observé. par exemple, eent cinquante crues annuelles bien distinctes.

On peut, simplement, dire qu'on a ainsi une borne superieure de l'information.

2" Par suite du fait que les phénomenes météorologiques sont beaucoup moins fortement corrélés que les débits, on gagnerait sans doute notablement plus d'incormation en appliquant l'idée de M. Durraut aux pluies et non aux débits, a condition de disposer de séries d'observations suffisamment riches. On serait conduit it une utilisation statistique de la méthode connue sous le nom de "transposition des averses ».

Bien entendu, une telle méthode suppose nécessairement l'analyse du mécanisme de formation des crues it partir des pluies, sur le cours d'eau que l'on étudie.

II. Durfaut confirme l'intérêt d'operer sur des crues independantes et, pour distinguer celles-ci, de connaître leur structure météorologique, et, notamment, quelle est, en fonction de son extension géographique et de sá durée, la vitesse de pluie d'une probabilité déterminée.

Cette remarque conduit M. Mondat à souligner l'intérêt diroir des relevés très précis d'intensité d'averses.

M. le Président remarque que les valeurs de M. Durraut, basées sur le facteur humain, sont valables pour la France continentale, mais non pour certains pays outre-mer où les facteur's économiques sur lesquels M. Danes a insisté avec raison seraient prépondérants; en Algérie, par exemple, pour deux grands aménagements en cours (Iril Emda et Djen-Djen en Kabylie) le risque an point de vue populations est restreint, alors iue les conséquences de ruptures des barrages de ces aménagements seraient telles qu'elles compromettraient gravement l'économie algérienne au point de vue énergétique.

M. le Président conclut que la question soulevée pal M. Dufraut est loin d'ètre épuisée et devra être reprise devant la Commission pour la determination des débits de crue, ainsi d'ailleurs qu'une étude mathématique de M. MaInLET d'Alger sur le même problème.

M. le Président remercie M. Duffaut. 